one ever found and of great importance in showing the form of the sexual phase, as it will now be possible to search for a known fixed form.

It is thus firmly maintained, supporting Winge (1923) and others, that "the pelagic Sargassum of the Sargasso Sea, apart from its marginal zones, is probably an essentially self-sustaining unit with a potentially infinite longevity and a very long normal expectancy of life for its individual plants, receiving (at most) only a very slight annual contribution from benthonic vegetation merely sufficient to compensate for its own loss by a slight excess of mortality over vegetative growth increment, suffered mainly through a continually occurring chance return of some of its members to the dangerous marginal zones from which a corresponding recruitment of freshly detached plants which have escaped its hazards are received in exchange."

The significant fact remains that no fixed benthonic stage is yet known of either of the two predominant types of floating Sargassum weed of the Sargasso Sea, namely, $S$. natans and $S$. fuitans. It seems that it must be concluded that here we have an essentially self-sustaining community of plants in which the pelagic existence has become secondarily normal, and which are able to maintain and multiply themselves indefinitely by mere vegetative growth, mechanical fragmentation and irregular partition of the individuals through disintegration from the basal end.

\title{
RECENT AMERICAN INVESTIGATIONS IN EMBRYOLOGY
}

$\mathrm{A}^{\mathrm{N}}$ $\mathrm{N}$ interesting paper of practical importance in orthopædic practice, entitled "The Functional Results of Muscle Transposition in the Hind Limb of the Rat", was read by Prof. R. W. Sperry at the Columbus Meeting of the American Association last December. The muscles operating the hind foot of this animal were transplanted in reverse, with the result that both reflex and voluntary movements of the hind limb were also reversed. Motion pictures showed that even after a year's trial of the new arrangement, there was no adjustment, and although the muscles worked the limb, the motor nerve patterns for limb co-ordination were on the old basis. Unfortunately the author does not state the names or number of the transplants.

Papers on experimental embryology related to the early development of the sea urchin, Arbacia punctulata. Profs. W. C. Allee and Asher J. Finkel, starting from the observed acceleration in cleavage-rate due to density of egg population and suspecting that some growth-promoting substance was responsible, carried out a series of experiments at Woods Hole with the intention of finding out more about the stimulating factor. They did not succeed in determining the chemical nature of the substance or substances; but their experiments showed that an extract from Arbacia eggs, even in "extremely dilute concentrations, can accelerate the early development of Arbacia eggs to a significant degree over that of untreated but otherwise similar eggs". They suggest that "undercrowding is as real in its effects as overcrowding".

Miss Ethel Browne Harvey studied the role of the nucleus and the cytoplasm in Arbacia eggs. Unfertilized eggs, broken into half eggs by centrifugal force, give a nucleated and a non-nucleated half. Both halves can be fertilized, or activated artificially. In the former case two plutei are produced; in the latter, the nucleated half becomes a pluteus, the nonnucleated half a blastula only. Fertilized eggs behave differently. A half containing both pronuclei forms a blastula, or at most a defective pluteus. A nonnucleated half, although its cytoplasm is affected by fertilization, does not even cleave, but the cytoplasm may make an atternpt to produce asters.

The author calls the development of the nonnucleated half of the artificially activated but unfertilized egg "parthenogenetic merogony". But since this half develops to the blastula stage only, it would appear that a nucleus is essential for the differentiation of the cells, though not for their mere multiplication.

Prof. Robert Chambers and E. L. Chambers found that fertilization of the egg in Arbacia could be arrested by immersing the egg immediately after insemination in a solution of potassium chloride, the strength of which is not stated. The return of the eggs so treated to normal sea-water more than an hour later causes resumption of activity of the sperm and subsequent normal development.

Two papers on transplantation of embryonic limb buds in birds were read by Prof. H. L. Eastlick. In the first set of experiments the chick embryo was the host, and duck, chukar partridge, quail, guinea fowl and turkey were the donors of the grafts. Both leg and wing were employed, transplantation being effected on embryos two to three days old. The egg was then re-sealed and put back in the incubator for further development. Fifteen per cent of the embryos hatched with the graft as a fifth limb. These grafts retained the structural characters of the donor species, except in a few cases where a partial merging of skin characters of donor and host occurred. 'Thus a duck leg graft formed a webbed foot, the feathers of which failed to develop after a poor attempt to do so ; they were followed by a second growth of host feathers. In other cases the graft limb grew at first but was later resorbed.

According to a second paper the birds mentioned above were used as hosts, but did not prove as successful for this purpose as the chick. Although the graft retained many of the characters of the parent bird, the age and extent of the graft affected the result. In particular, a graft removed with a portion of the neural crest developed feathers of the same colour as the graft's parent, whereas a graft cut laterally so as not to include the neural crest partook of the colour characters of the host. Grafts from white silky bantams, the feathers of which have no hooklets on the barbules, retained this character when transplanted to White Leghorn or Barred Plymouth Rock hosts. Prof. Eastlick described and illustrated some of his results in a brief communication published in Nature of August 26, 1939, p. 380. 